\title{
Article \\ Assistance of a Person with Muscular Weakness Using a Joint-Torque-Assisting Exoskeletal Robot
}

\author{
Hyunjin Choi
}

Citation: Choi, H. Assistance of a Person with Muscular Weakness Using a Joint-Torque-Assisting Exoskeletal Robot. Appl. Sci. 2021, 11, 3114. https://doi.org/10.3390/ app11073114

Academic Editor: Manuel Armada

Received: 17 February 2021

Accepted: 29 March 2021

Published: 31 March 2021

Publisher's Note: MDPI stays neutra with regard to jurisdictional claims in published maps and institutional affiliations.

Copyright: (C) 2021 by the authors Licensee MDPI, Basel, Switzerland. This article is an open access article distributed under the terms and conditions of the Creative Commons Attribution (CC BY) license (https:// creativecommons.org/licenses/by/ $4.0 /)$.
Department of Human Intelligence Robot Engineering, Sangmyung University, Cheonan 31066, Korea; hyunjin@smu.ac.kr

\begin{abstract}
Robotic systems for gait rehabilitation have been actively developed in recent years; many of the rehabilitation robots have been commercialized and utilized for treatment of real patients in hospitals. The first generation of gait rehabilitation robots was a tethered exoskeleton system on a treadmill. While these robots have become a new trend in rehabilitation medicine, there are several arguments about the effectiveness of such robots due to the passiveness of the motions that the robots generate, i.e., the continuous passive motions may limit the active involvement of patients' voluntary motion control. In order to let a patient be more actively involved by requiring the self-control of whole-body balance, untethered powered exoskeletons, wearable robots that patients can wear and walk on the ground, are receiving great attention. While several powered exoskeletons have been commercialized already, the question about their effectiveness has not been cleared in the viewpoint of rehabilitation medicine because most of the powered exoskeletons provide still continuous passive motions, even though they are on the ground without tethering. This is due to their control strategy; the joints of a powered exoskeleton are position-controlled to repeatedly follow a predefined angle trajectory. This may be effective when a wearer is completely paraplegic such that the powered exoskeleton must generate full actuation power for walking. For people with muscular weakness due to various reasons, the powered exoskeleton must assist only the lack of muscular force without constraining human motion. For assistance and rehabilitation of people with partial impairment in walking ability, Angel Legs is introduced in this paper. The proposed powered exoskeleton system is equipped with a transparent actuation system such that the assistive force is accurately generated. The overall design and control of Angel Legs are introduced in this paper, and a clinical verification with a human subject is also provided.
\end{abstract}

Keywords: powered exoskeleton; gait rehabilitation; torque-assist control

\section{Introduction}

Walking that is significantly different from a normal gait is called an abnormal gait. Abnormality of walking can be generated by various causes, such as muscular weakness, paralysis, pain, limited range of motion, neurological disorders, and leg length discrepancy. Diseases that can cause abnormal gait include stroke, cerebral palsy, and Parkinson's disease. For example, after a stroke, most patients experience weakness or paralysis on one side of the body, and many of them also experience spasticity [1]. Due to the weakness of distal muscles, hemiplegic patients drag their legs in a semicircle. Additionally, patients with Parkinson's disease have a shuffling gait due to basal ganglia dysfunction. They may experience freezing at the beginning of their walk or may have a festinating gait during walking. Another representative cause of abnormal gait is cerebral palsy. Abnormal gait due to cerebral palsy includes equinus gait, crouch gait, jump knee, and drop foot or a simultaneous combination of these symptoms.

Therefore, abnormal walking occurs in the event of an abnormality in the sensory and motor systems that activate certain muscles. If the Hamstring weakens, a hyperextension of knee occurs in the stance phase and the knee is not flexed in the terminal swing and 
continues to extend. Furthermore, if there is an abnormality in hip abductor muscles, gluteus medius gait occurs, in which the body leans over the opposite leg during the swing phase. Joint pain and weakness due to aging also cause abnormal walking. Frank et al. showed that muscular strength declines with age and decreases by up to $50 \%$ by the age 70 [2]. Patients with abnormal walking and balance disorders are afraid of walking and cannot walk for a long time. These mobility disabilities increase the risk of dependency in physical activities in daily living [2,3].

If abnormality is not considerable, balancing can be assisted by a simple walking aid such as canes, walkers, crutches, and so on. Using these, the base of support can be widened to help maintain balance [4]. However, when used for a long time, the arm muscles may become painful. In some cases, an Ankle Foot Orthosis (AFO) or a Knee Ankle Foot Orthosis (KAFO) is used to companstate weakness. There are various materials and structures of orthosis; recently, electrical devices that incorporate active joints have been introduced [5].

For people with partial impairment in walking ability, it is mandatory to stand up and perform walking motions frequently with the help of assistive devices (e.g., a harness system, a robotic rehabilitation treatment system, etc. [6]) to maintain health conditions. In this respect, wearable robots enabling the patients with paretic gait walk on the real ground can be used. These robots are effective both for rehabilitation and for an increase in the patients' mobility.

In recent years, wearable robots for gait assistance have been developed and commercialized [7]. Among many applications of the wearable robots, ones for assisting people with complete paraplegia may be the mostly popularized. While wheelchairs can improve the mobility of patients with complete paralyses, they cannot provide standing postures and bipedal walking, which are important for healthcare. Many studies have shown that bipedal walking training can increase quality of life and life satisfaction [6,8-10]. ReWalk, a commercial robot by ReWalk Robotics [11], is currently being distributed to rehabilitation hospitals around the world. Other commercially available robots for disabled and paralyzed people are Ekso [12] from Ekso Bionics, Indego [13] from Parker Hannifin corporation, and Rex [14] from Rex Bionics. Many other robotic exoskeletons are being developed and commercialized $[15,16]$.

In such cases of a wearable robot for complete paraplegics, i.e., people with complete loss of walking ability, it is common to use a position tracking control scheme. There are cases where position tracking control is used in robots for people with partial impairment in walking ability, such as hemiplegic or stroke patients [17]. This allows the user to imitate the trajectory of a normal gait or the trajectory of a healthy side leg. However, for incomplete paraplegics with remaining sensory and motor functions, the position control strategy constrains the human's voluntary motion, and thus, the wearers of such robots have to learn to suppress voluntary motion and to fully rely on the robot. This may not be appropriate in the viewpoint of rehabilitation medicine, and at present, it is difficult to find a wearable robot that can compensate only for the lack of the weakness of muscular power without disturbing a human's voluntary motions. There are soft wearable suits, but such soft a mechanism is not suitable to help self-balancing of people with muscular weakness $[6,18]$.

For gait rehabilitation and assistance of people with partial impairment of walking ability, a torque control method may be more effective. The torque control is a method that allows the user to control the movement while allowing the appropriate assistance at the required moment, such as pushing the swing from behind. Therefore, there is a clear need for a control method that captures the exact moment when assistance is needed and provides as much assistive torque as needed.

The main aim of this paper is to improve the walking of patients with paretic gait by applying the torque assist algorithm using a wearable robot. In order to achieve this goal, the following objectives are formulated:

- integration of torque-mode actuators and a wearable robot, 
- development of a real-time gait detection algorithm with simplified gait phases,

- generating a patient-customized assistive torque and control algorithm, and

- validation of the effect of walking assistance using the proposed wearable robot through a case study.

\section{Angel Legs: A Powered Exoskeleton with Torque-Mode Actuators}

Parts of this section have been previously presented in [19].

In this paper, Angel Legs, the powered exoskeleton shown in Figure 1, is introduced. The proposed robot has been particularly designed for assisting people with partially impaired walking ability. In recent years, assistive robots for people with partially impaired walking ability are being commercialized by a few research groups. Cyberdyne Inc., Tsukuba, Japan, a popular wearable robot company in Japan, has developed the HAL series. ReWalk has launched a soft exo suit, called ReStore, for assisting stroke patients [20]. The existing products, however, may not yet provide a practical and attractive solution to real-world users because they require attachment of electric sensors on the nude skin and impose a large resistance on the human joints due to actuator friction. Robotic assistance with a large actuator friction may not be the right solution for people with partially impaired walking ability. The soft exo suit is very promising in this aspect, but it is not suitable to help maintain the whole-body balance of a patient due to the characteristics of the soft mechanism. Considering these aspects, Angel Legs has been developed based on series elastic actuation (SEA) systems, which enables accurate assistance with almost-zero mechanical impedance [21], i.e., "transparent assistance".

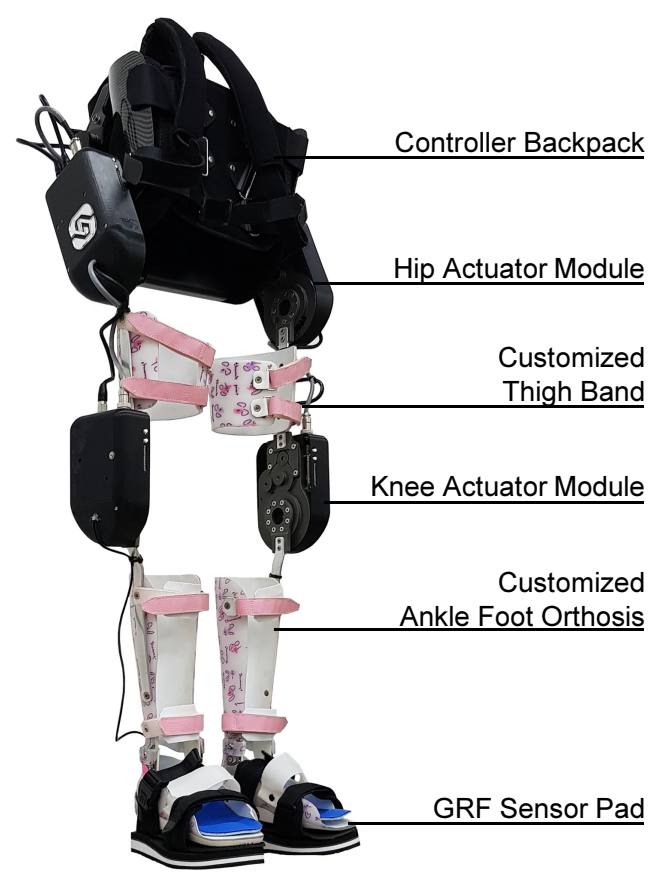

Figure 1. Angel Legs, a lower-extremity powered exoskeleton for assisting joint torques [19].

The design of Angel Legs demands unique requirements, such as minimal mechanical impedance and high back-drivability, as well as high power density. The mechanical parts must be ergonomically designed, such that humans can use the devices for a long period of time without discomfort. The motor control function of people with impaired walking ability is not as robust as that of the normal, and thus, the overall human body system becomes vulnerable to disturbances (i.e., external forces, inclinations, etc.) or model variations (i.e., loads, etc.). It is, however, still active, unlike complete paraplegic patients, and thus, the robot must not generate any unexpected resistance against the voluntary motions of people with impaired walking ability for safety and for minimal discomfort. Therefore, both the design and control of assistive robots for people with 
partially impaired walking ability are very challenging. As the muscular strength of the target users is weakened (but not fully impaired), the overall weight, the actuation resistance and precision, and the comfort of the robot hardware have always been the main concerns in the mechanical design of Angel Legs.

\subsection{Power-Pack System}

Angel Legs includes a high capacity battery pack made of lithium-ion cells. The nominal voltage of the battery pack is $44 \mathrm{~V}$, and the energy capacity is $3 \mathrm{Ah}$, which is good for assisting walking for about two hours. To provide a large electric current to the electric motors, in particular, to initiate motion at a stall state, battery cells with a high C-rate are utilized. The nominal voltage is selected at $44 \mathrm{~V}$ because the relevant international standard (e.g., ISO13482) states that a voltage under $50 \mathrm{~V}$ is regarded as safe.

The battery pack can be replaced easily. When the battery pack is fully discharged but the user wants to continue activities without recharging the battery, it can be disassembled by simply opening two latches placed on both sides of the battery pack and can be replaced with a spare battery pack. The battery pack is controlled by a high-performance battery management system (BMS). The functions of the BMS include (1) over-charge and overdischarge protection, (2) over-current protection, (3) electric short protection, (4) battery cell temperature monitoring and control, and so on. In addition, the BMS balances the remaining energy in each of the battery cells, such that all the battery cells can be equally charged and discharged. The BMS frequently reports the status of the battery pack to the main processor (i.e., NI sbRIO-9651) via the I2C communication. The remaining battery energy is displayed at the main control panel.

\subsection{Actuation System of Angel Legs}

An actuation module of Angel Legs, called Angel Joint v1, is shown in Figure 2. For accurate control of the assistive torque transferred to the human joint, a structural spring is utilized, as shown in Figure 2b, which realizes the series elastic actuation. The spring plays the roles of an energy buffer and a torque sensor [22,23]. By measuring the spring deflection, the assistive torque can be measured. Therefore, the precise torque control can be achieved by controlling the spring deflection. In addition, the spring is helpful in improving the safety of the actuation module.

Angel Joint v1 consists of a brush-less direct-current (BLDC) motor (Maxon motor company, $70 \mathrm{~W}$, EC45-flat), spring-embedded gears, sensors for measuring the spring deflection, as well as the absolute angle of the human joint. However, the actuation power is not very meaningful because the motor is operated at low speeds, where the stall state takes a place frequently. After the gears and a spring, the magnitude of an assistive torque is about $14.2 \mathrm{Nm}$ in a continuous assistance condition and $35.5 \mathrm{Nm}$ in a short-term assistance condition. The other mechanical specifications are shown in Table 1.

The actuation performance of Angel Joint v1 is determined by the design of the springembedded gears as well as a control algorithm controlling the spring deflection. As shown in Figure 2b, Angel Joint v1 is designed to have two spring-embedded gears. For the sake of minimal weight and volume, the "spring-embedded" gears embed springs, such that the gears exhibit elasticity without any additional springs. As the spring-embedded gears should be made of a strong material to prevent the failure of gear teeth, the spring constant is unavoidably high. Therefore, Angeloint v1 has two spring-embedded gears to realize the desired spring constant. 


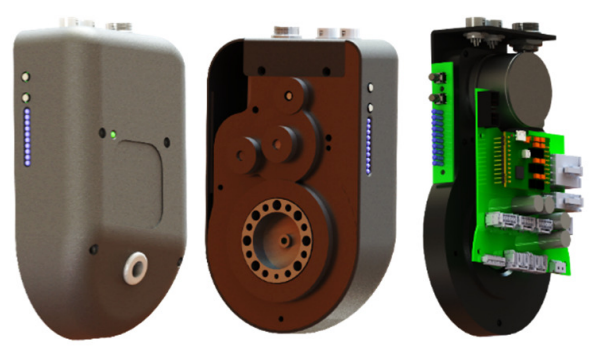

(a)

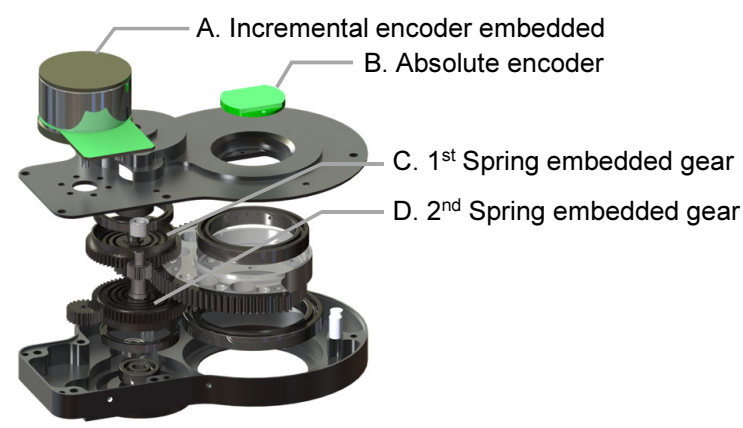

(b)

Figure 2. AngelJoint v1: (a) the outside view and (b) the structure of the series elastic actuation system.

Table 1. Specification of Angel Joint v1.

\begin{tabular}{ccc}
\hline Spec & Unit & Value \\
\hline Dimension & $\mathrm{W} \times \mathrm{D} \times \mathrm{H}[\mathrm{mm}]$ & $110 \times 180 \times 65$ \\
Weight & {$[\mathrm{kg}]$} & 0.76 \\
Assistive torque & {$[\mathrm{Nm}]$} & 14.2 (cont.), 35.5 (peak) \\
Maximum speed & {$[\mathrm{rpm}]$} & 66.4 \\
Range of motion & {$[\mathrm{deg}]$} & 170 \\
\hline
\end{tabular}

Figure 3 shows the linearity of springs in the Angel Joint. It was measured by a dynamometer. The spring constant was measured by $302.34 \mathrm{Nm} / \mathrm{rad}$ and the backlash, i.e., the dead-zone at the zero-torque shown in Figure 3 was measured as 0.14 degrees (i.e., $0.0024 \mathrm{rad}$. The backlash was caused by the tolerance of gear teeth, and the torque measurement error due to the backlash was about $0.74 \mathrm{Nm}$, i.e., $302.34 \mathrm{Nm} / \mathrm{rad} \times 0.0024 \mathrm{rad}$. This error is small enough compared with the magnitude of desired assistive torque (i.e., 14.2 Nm).

\subsection{Torque Control Algorithm}

One of the most unique characteristics of Angel Legs is the "transparent" actuation, i.e., precise torque control without resistance. The transparent actuation is realized by the control of the spring deflection in a series elastic actuator. Figure 4 shows the control block diagram of the transparent actuation. The desired (i.e., reference) assistive torque $\left(\tau_{d}\right)$ is loaded when the user selects the operation mode through user interface systems, such as a tablet device and an embedded controller button. By Hooke's law, $\tau_{d}$ is then converted into the desired angular position of the motor $\left(\theta_{m d}\right)$, i.e., $\theta_{m d}=\theta_{h}+k^{-1} \tau_{d}$, where $\theta_{h}$ and $k$ are the human joint angle and the spring constant, respectively.

The encoder signals (i.e., a pair of an absolute encoder and an incremental encoder for each actuation module (see A and B in Figure 2b)) are utilized for measuring $\theta_{m}$ and $\theta_{h}$. As the human joint angle may include a measurement error due to the inherent characteristic of a magnetic encoder, $\theta_{h}$ is compensated by a pre-obtained compensation signal (see (a) in Figure 4). 


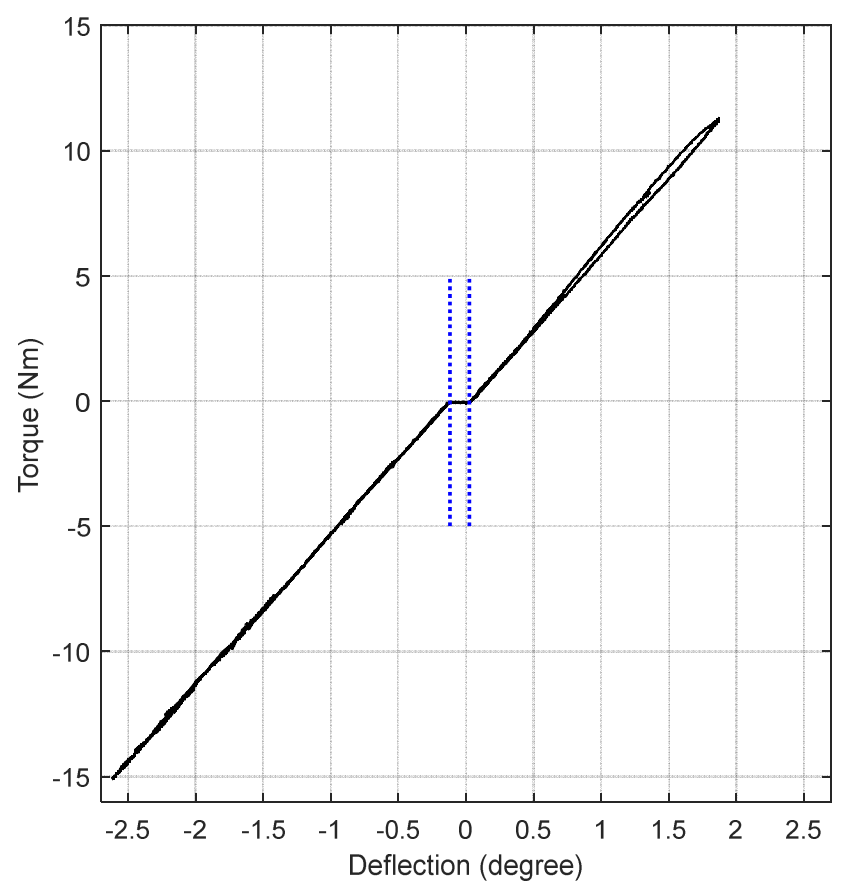

Figure 3. Linearity of spring embedded in gears.

The motor angular position can be accurately controlled to follow $\theta_{m d}$ based on the position control method. In Angel Joint v1, a proportional-integral-derivative(PID) controller (see (b) in Figure 4) and an impedance compensator (see (d) in Figure 4) are implemented for position control. The PID controller gains are changed according to the magnitude of the torque error (see (c) in Figure 4).

Once the motor is accurately controlled, the spring deflection is accurately controlled, which implies the accurate control of assistive torque.

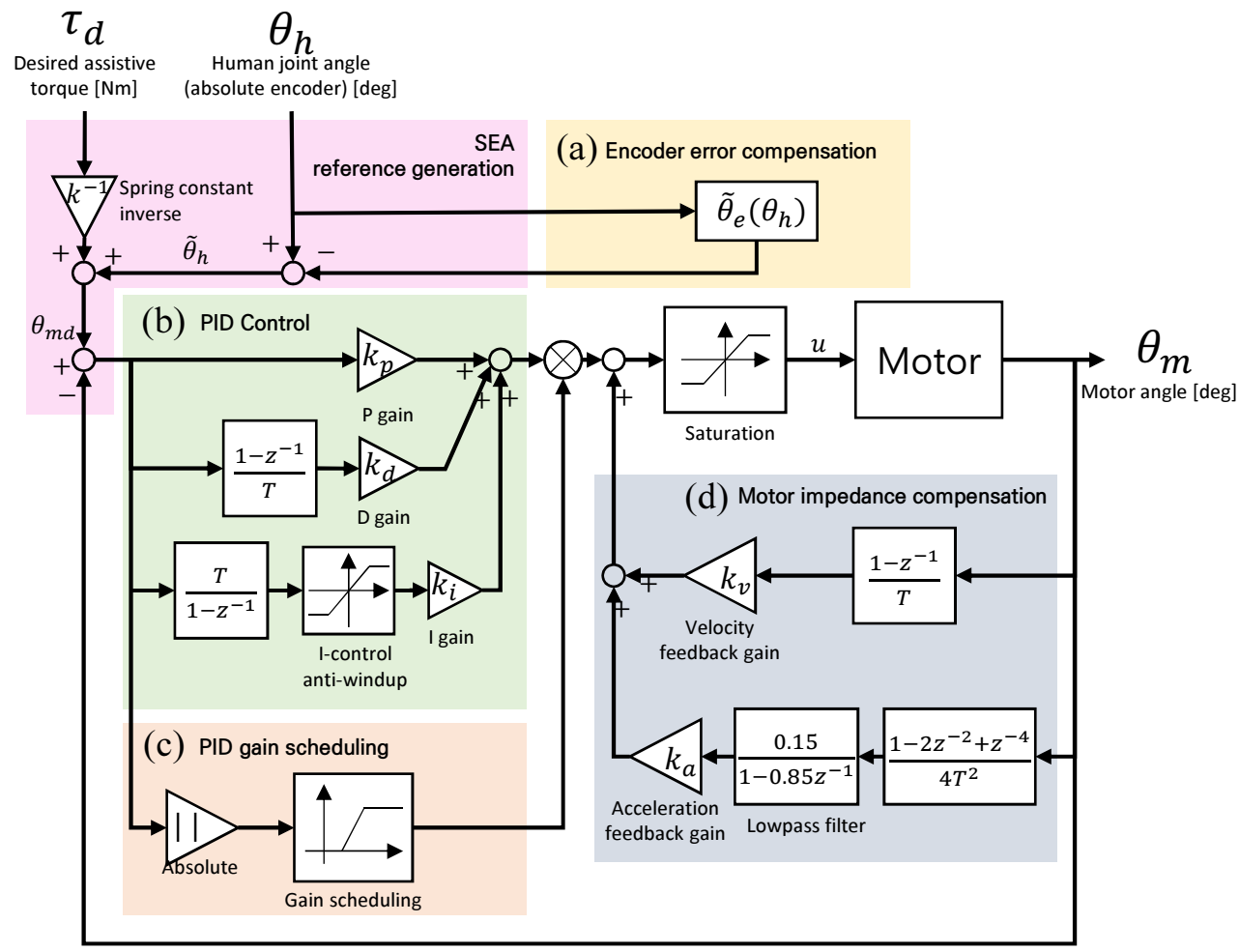

Figure 4. Assistive torque control of the series elastic actuation (SEA) module. 


\section{Real-Time Gait Detection Method}

\subsection{Classification of Gait Phases}

Human walking can be regarded as a periodic movement composed of several phases. Using insole sensors with two sensors on a foot (i.e., toes area and heel area), four phases can be detected; heel strike, mid stance, terminal stance, and swing. However, if the leg muscles or joints are weakened or damaged, the sub-phases of the stance phase (i.e., heel strike, mid stance, and terminal stance) may not be obvious in gait analysis. For example, patients with equinus gait (i.e., tiptoe gait) do not touch their heel to the ground during walking. In this case, a gait can be classified into two main stages: (1) a stance phase in which any part of the foot touches the ground and (2) a swing phase in which the foot is completely off the ground. Figure 5 shows the simplified gait phases of a leg. Considering the phase of the opposite leg, the stance phase of the leg can be divided into the first double limb support phase (DS1), single limb support phase, and the second double limb support phase (DS2). Therefore, the gait can be classified into four index phases as follows.

- Gait index 1: Double limb support with the right leg forwarded (DS1)

- Gait index 2: Single limb support of the right leg (left swing)

- Gait index 3: Double limb support with the left leg forwarded (DS2)

- Gait index 4: Single limb support of the left leg (right swing)

The double limb support phase of the right leg includes the stance phase of the left leg, and the single leg support phase of the right leg means the swing phase of the left leg. Therefore, the proposed four-level classification covers the entire gait stage of both legs. People with paretic gait generally have longer double limb support times than healthy people. Except for those who are severely paralyzed or lack muscle strength enough to be unable to lift their legs at all, people walk in sequential phases when walking forward.

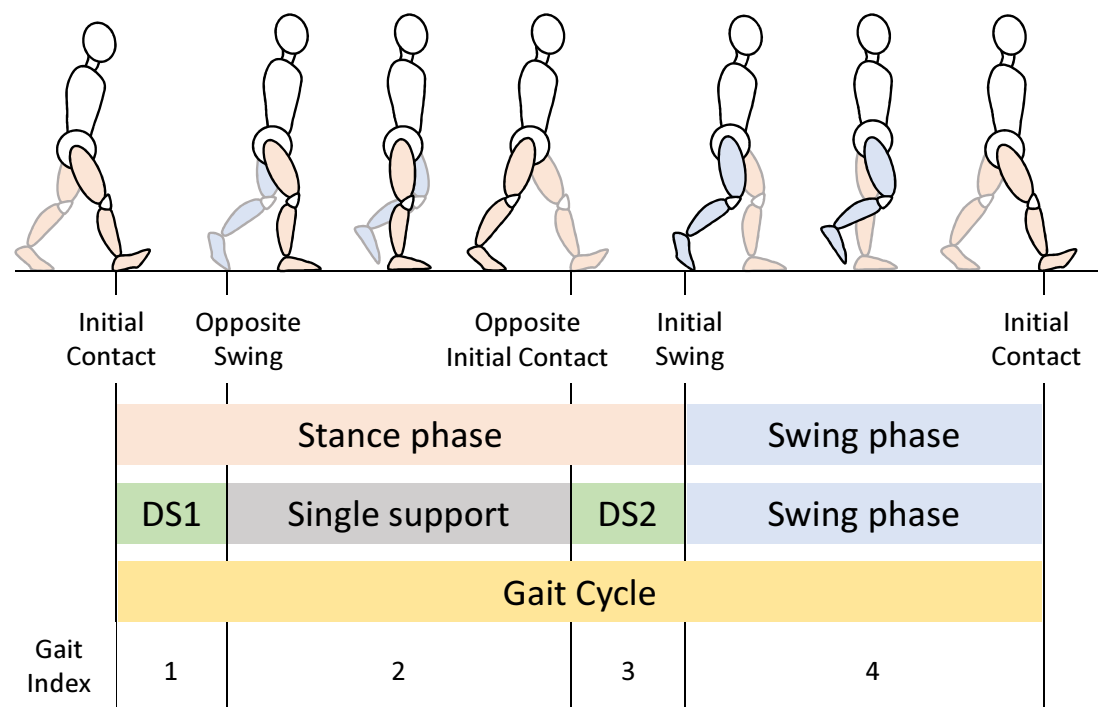

Figure 5. Gait patterns of a leg: one gait cycle is from when the foot initially contacts the ground to when the foot contacts the ground again. DS1 and DS2 mean the first and the second double limb support phases, respectively.

\subsection{Gait Detection Algorithm}

In this section, the gait index derivation from the measurement of the foot sensor-pad during walking is introduced. In the case of people with paralytic gait, the swing motion is performed only when the center of gravity is stable. Therefore, the timing of the initial swing depends on the intention of the person. Robotic support for incomplete paraplegics should also be applied when the person intended. Otherwise, the operating force will cause unstable movement of humans and robotic systems. In this paper, the walking intention is monitored in real-time through the gait index analysis method using the foot sensor pad. 
Swing phase of a leg can be determined as $\mu_{\text {swing }}$ :

$$
\mu_{\text {swing }}(t)= \begin{cases}1, & \text { if } f_{\text {toe }}(t)<s \text { and } f_{\text {heel }}(t)<s \\ 0, & \text { otherwise }\end{cases}
$$

where $f_{\text {toe }}$ and $f_{\text {heel }}$ are the measurements of the toe and heel sensor pads, respectively, and $s$ is the threshold to check that the sensor is pressed. Figure 6a shows the swing phases of both leg while walking, and $\mu_{\text {swing }}=0$ represents the stance phase. As described in Section 3.1, the gait can be classified into four phases. To switch the control scheme according to the gait phase, it is necessary to detect the moment of phase change.

The falling edge of $\mu_{\text {swing,right }}$ represents the initial contact event of the right leg, which is equivalent to Gait index $=1$, the rising edge of $\mu_{\text {swing,left }}$ is equivalent to Gait index $=2$, the falling edge of $\mu_{\text {swing, left }}$ is equivalent to Gait index $=3$, and the rising edge of $\mu_{\text {swing,right }}$ is equivalent to Gait index $=4$. The derived gait index of walking is shown in Figure $6 \mathrm{~b}$.

(a)

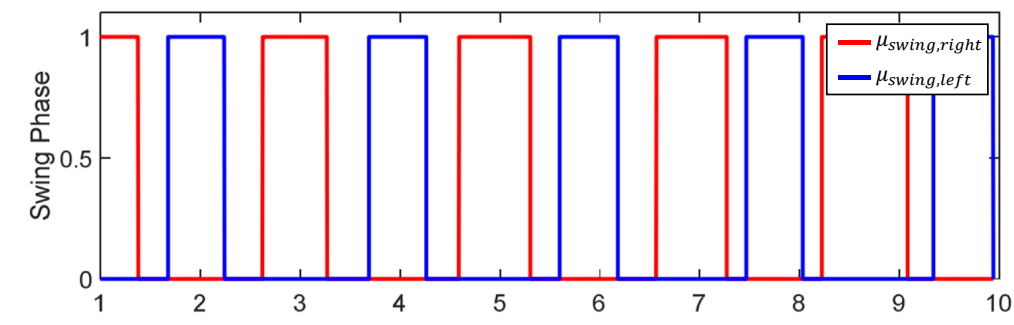

(b)

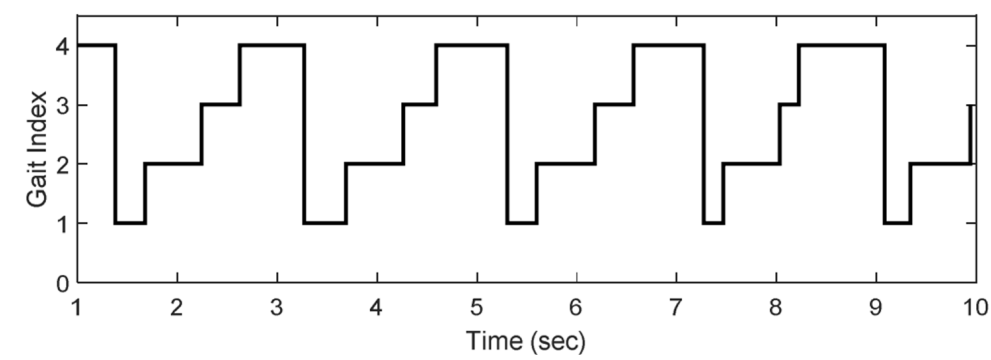

Figure 6. Gait index measurement results: (a) swing phases of both legs. Values of 1 represent the swing phase and values of 0 represent the stance phases. (b) Representative gait index.

\section{Joint Torque Assistance Control}

\subsection{Assistive Control}

As the actuation modules can generate assistive torques accurately, Angel Legs is able to assist the human motion following the "assistance as needed" strategy [24]. Figure 7 shows the overall assistance control method of Angel Legs. The gait intention detection algorithm makes a triggering signal according to the ground reaction force(GRF) signals and the hip and knee joint angles. Then, the desired assistive torque is generated according to either (1) a look-up table or (2) a feedback algorithm, which should be selected depending on the assistance strategy for each individual user. The desired assistive torque is transferred to the torque control algorithm in Figure 7 and then the user is assisted with the actual assistive joint torque. 


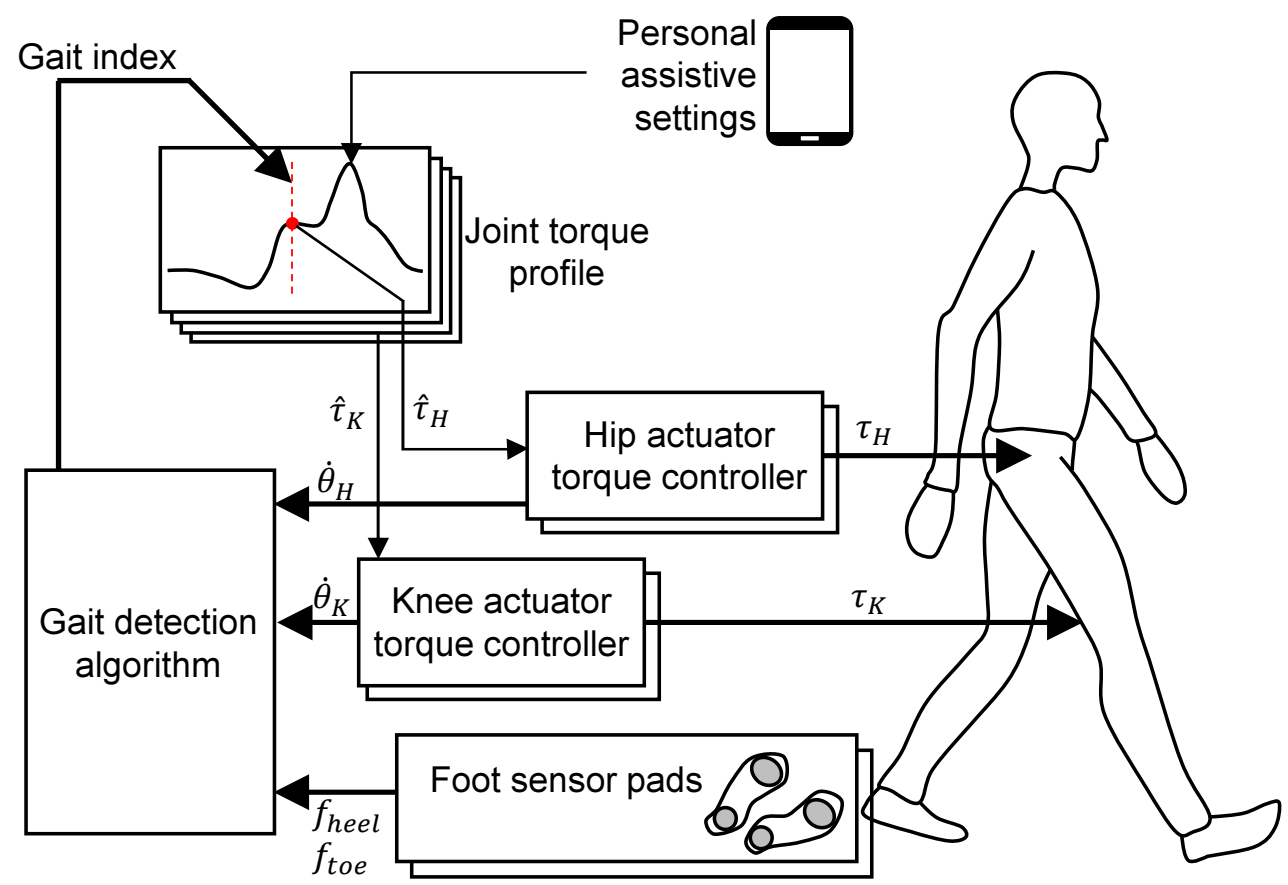

Figure 7. The overall control structure of Angel Legs.

The assistance parameters should be adjusted for each individual user. Angel Legs can provide more natural assistance if adjusted for each user. The personalized assistance modes, the level of assistance, the range of motion of joints, etc. can be adjusted. The example shown in the Figure 8 is the predefined torque profile (i.e., the look-up table method). Through the tablet computer, the user can adjust the instances and magnitudes of peak torques, and then, the remaining parts are automatically interpolated to prevent any discontinuous or impulsive torque.

\subsection{Design of an Assistive Joint-Torque Profile}

Although human motion is not generated by a rotary actuation on a single axis joint, the sagittal joint torques (i.e., joint moments) can be estimated by using inverse dynamics. During stance phases, both hip and knee joints need extension torques to resist and propulsion, and flexion torques required to push forward the swing side leg. People with incomplete paraplegia have a lack of muscle strength, making it difficult to push the forelimbs forward, and have a short swing period. Therefore, once the initial swing is detected, the assistive torque should be provided for an appropriate amount of time so that the wearer can use the assistive torque through repeated practice.

For individual gait training, it is necessary to easily modify the assistive torque profile without compromising the continuity of the trajectory function. Therefore, polynomial functions were used in this research to find the equations for joint torque trajectories. In addition to the conditions mentioned above, the derivatives at the beginning and end of the gait should be zero in order to smoothly switch to other motions such as standing. No other inflection points should exist between the main points. Therefore, we divided the trajectory to four sections based on the main points, and the third order polynomials were derived for each section.

While $p_{i}=\left[\begin{array}{l}x_{i} \\ y_{i}\end{array}\right]$ means the representative point of the torque trajectory, where $x_{i}$ is the percentage of gait cycle and $y_{i}$ is the reference torque, the coefficients of polynomials can be derived as below.

$$
\left[\begin{array}{c}
y_{i} \\
y_{i+1} \\
0 \\
0
\end{array}\right]=\left[\begin{array}{cccc}
x_{i}^{3} & x_{i}^{2} & x_{i} & 1 \\
x_{i+1}^{3} & x_{i+1}^{2} & x_{i+1} & 1 \\
3 x_{i}^{2} & 2 x_{i} & 1 & 0 \\
3 x_{i+1}^{2} & 2 x_{i+1} & 1 & 0
\end{array}\right]\left[\begin{array}{c}
a_{i, 3} \\
a_{i, 2} \\
a_{i, 1} \\
a_{i, 0}
\end{array}\right]
$$


the inverse of which is

$$
\left[\begin{array}{l}
a_{i, 3} \\
a_{i, 2} \\
a_{i, 1} \\
a_{i, 0}
\end{array}\right]=\left[\begin{array}{cccc}
x_{i}^{3} & x_{i}^{2} & x_{i} & 1 \\
x_{i+1}^{3} & x_{i+1}^{2} & x_{i+1} & 1 \\
3 x_{i}^{2} & 2 x_{i} & 1 & 0 \\
3 x_{i+1}^{2} & 2 x_{i+1} & 1 & 0
\end{array}\right]^{-1}\left[\begin{array}{c}
y_{i} \\
y_{i+1} \\
0 \\
0
\end{array}\right]
$$

Therefore, the joint torque profile of hip joint can be expressed as

$$
f(x)=\left\{\begin{array}{lll}
a_{1,3} x^{3}+a_{1,2} x^{2}+a_{1,1} x+a_{1,0} & \text { if } \quad x_{1} \leq x<x_{2} \\
a_{2,3} x^{3}+a_{2,2} x^{2}+a_{2,1} x+a_{2,0} & \text { if } \quad x_{2} \leq x<x_{3} \\
a_{3,3} x^{3}+a_{3,2} x^{2}+a_{3,1} x+a_{3,0} & \text { if } \quad x_{3} \leq x<x_{4} \\
a_{4,3} x^{3}+a_{4,2} x^{2}+a_{4,1} x+a_{4,0} & \text { if } \quad x_{4} \leq x<x_{5}
\end{array}\right.
$$

Figure 8 shows the assistive torque profile based on the gait phases. The representative points $h_{i}^{\prime} s$ and $k_{i}^{\prime} s$, i.e., the maximum points of flexion and extension torques of hip and knee joints, can be individually set. The assistance instance is based on the users' gait phases. For the right leg, (a) in Figure 8 is the same as Gait index $=1$ (i.e., right contact), and (b) is the same as Gait index $=2$ (i.e., left toe-off). In this case, the time between (a) and (b) can not be predetermined since it truly depends on the users intent. When the user tries to move the hind leg for a swing motion, then Gait index $=2$ is detected and the assistive torque corresponding to those between (b) and (c) are applied. Although the time of the (c) is not exactly the same as the actual Gait index $=3$, which also depends on the user's intent, the same assistive torque of time (c) is applied until (d), i.e., Gait index $=4$. Similarly, the torque of (e) (i.e., Gait index $=1$ ) continues until the next gait event Gait index $=2$.
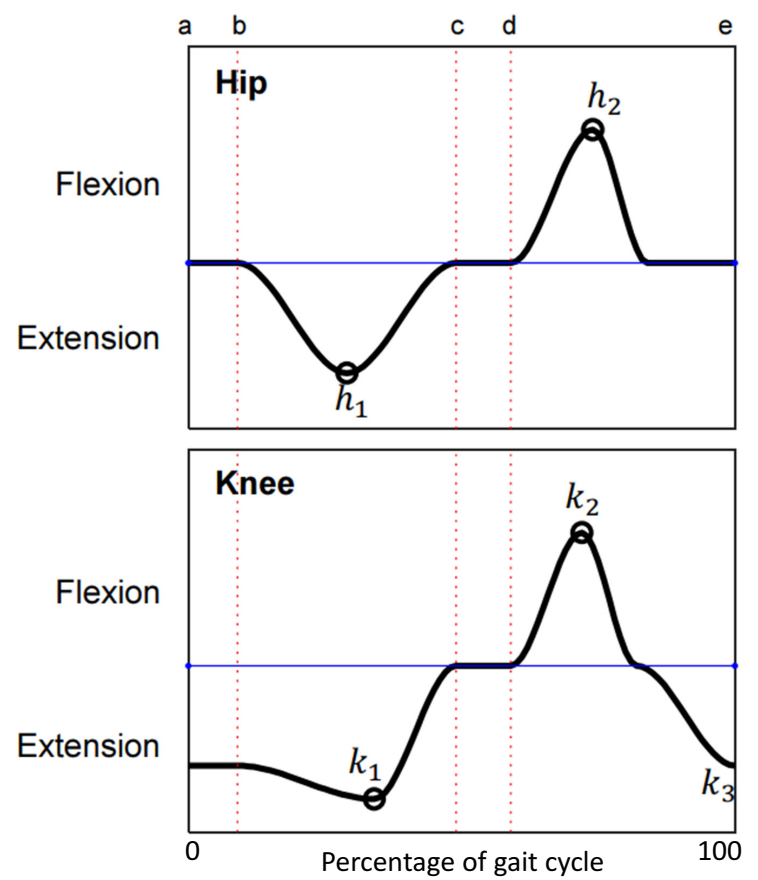

Figure 8. Assistive torque profile functions of hip and knee joints during a gait cycle: (a) the initial contact event of a leg, (b) the opposite foot-off event, (c) the initial contact of the opposite leg, (d) the foot-off (i.e., initial swing) event, and (e) the end of the gait (i.e., initial contact event of the next step).

The amplitudes of the peak torque can be adjusted depends on the patient's muscular strengths of each leg and desired assistance level. Based on the moments on normal gait with healthy people $[25,26]$, the peak moments for normal walking can be assumed as -0.5 and $1.2 \mathrm{Nm} / \mathrm{kg}$ for $h_{1}$ and $h_{2}$, respectively, and $-0.65,0.2$, and $-0.5 \mathrm{Nm} / \mathrm{kg}$ for $k_{1}, k_{2}$, and $k_{3}$, respectively. 


\section{Verification of Rehabilitation Effectiveness with a Case Study}

This section introduces a case study with Angel Legs. The participant in this paper was an 11-year-old female with incomplete paraplegia caused by spina bifida. In general, the muscle strengths of her lower extremities were grade $1 / 5$ to $3 / 5$ on the Medical Research Council scale. Figure 9 shows the scene of the motion capture-based gait analysis. The human subject was able to walk on the floor wearing solid ankle-foot orthoses. She had a large difference in left and right musculoskeletal functions and had to use trunk muscles to move her pelvis and legs. She also could not walk for a long time, so she used a wheelchair most of the time. Due to excessive pelvic anterior tilting caused by hip extensor weakness, the hip joints generally bent forward and showed little extension. During walking, the left and right hip joints could not be sufficiently stretched to the back and were bent by more than 20 degrees, which caused back pain when walking for a long time. Due to differences in left and right muscular strengths, there was a significant difference between the left and right sides in the stance periods and the range of motions of the knee. Since the right side muscle was weaker than the left side, the right stance period was shorter than the left stance period.

Parallel bars are the most commonly used method in the early stages of gait training for rehabilitation. This method helps patients become more confident about using walking equipment, such as crutches, canes, and walkers. In what follows, we describe the results of several assistive methods using Angel Legs, a torque-controlled wearable robot. Walking with parallel bars, wheeled walkers, and bilateral forearm crutches were attempted and then analyzed (Figure 10).

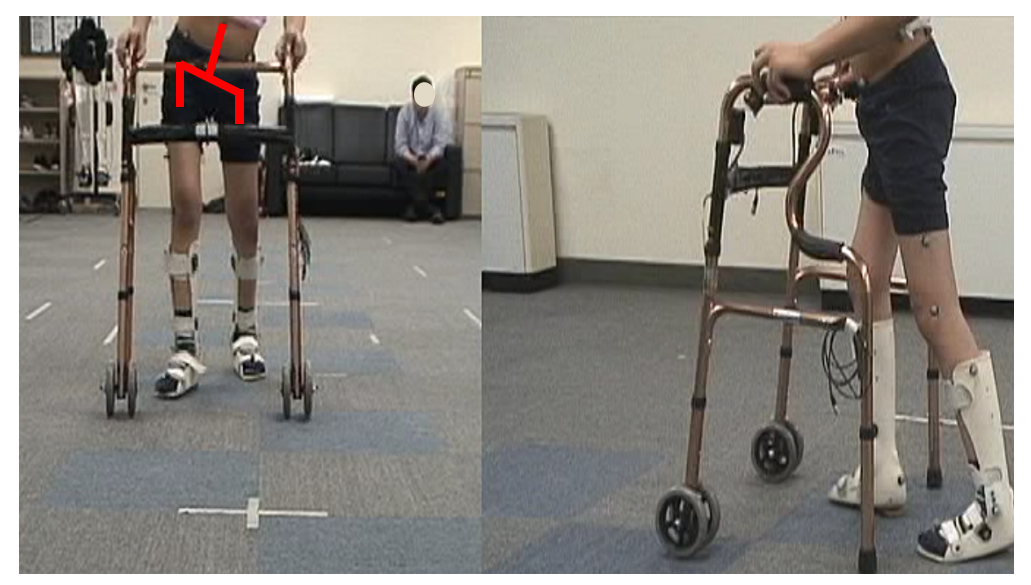

Figure 9. Gait analysis of a patient with spina bifida wearing Ankle Foot Orthosis (AFO) and using a walker.

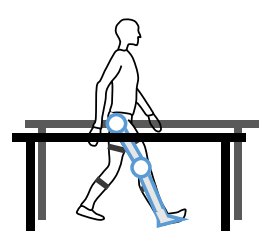

2 weeks

Parallel bar

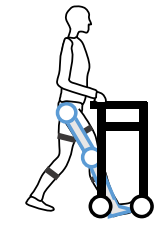

3 weeks Wheeled walker

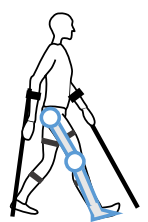

5 weeks

Bilateral crutch

Figure 10. Robotic gait training protocol.

\subsection{Assistance with Parallel Bars}

In the case of torque-based control, we applied the walking intention detection algorithm using insole pad sensors. The gait index was detected by the algorithm in real-time, and the robot provided the assist torque corresponding to the gait index. 
Figure 11 shows the kinematic results of the gait training with and without torque assistance of the robot on the parallel bars. In the case of walking using parallel bars, the typical camera-based motion capture system could not be used, since the body markers were blocked by parallel bars. Therefore, the gait information was acquired using the sensor system. Due to torque-based assistance, the difference in the left and right stance phases was reduced.

Equation (5) was used to determine the gait symmetry. Symmetry Index (SI) was defined as follows:

$$
S I=\frac{X_{L}-X_{R}}{0.5\left(X_{L}+X_{R}\right)} \times 100,
$$

where $X_{L}$ is the value of the non-injured side, which can be the left side in this case (i.e., the gait percentage of the stance phase), and $X_{R}$ is the value of the right side [27]. The gait symmetry index in the stance phase was improved from $15.07 \%$ without torque assistance to $-2.69 \%$ with torque assistance.
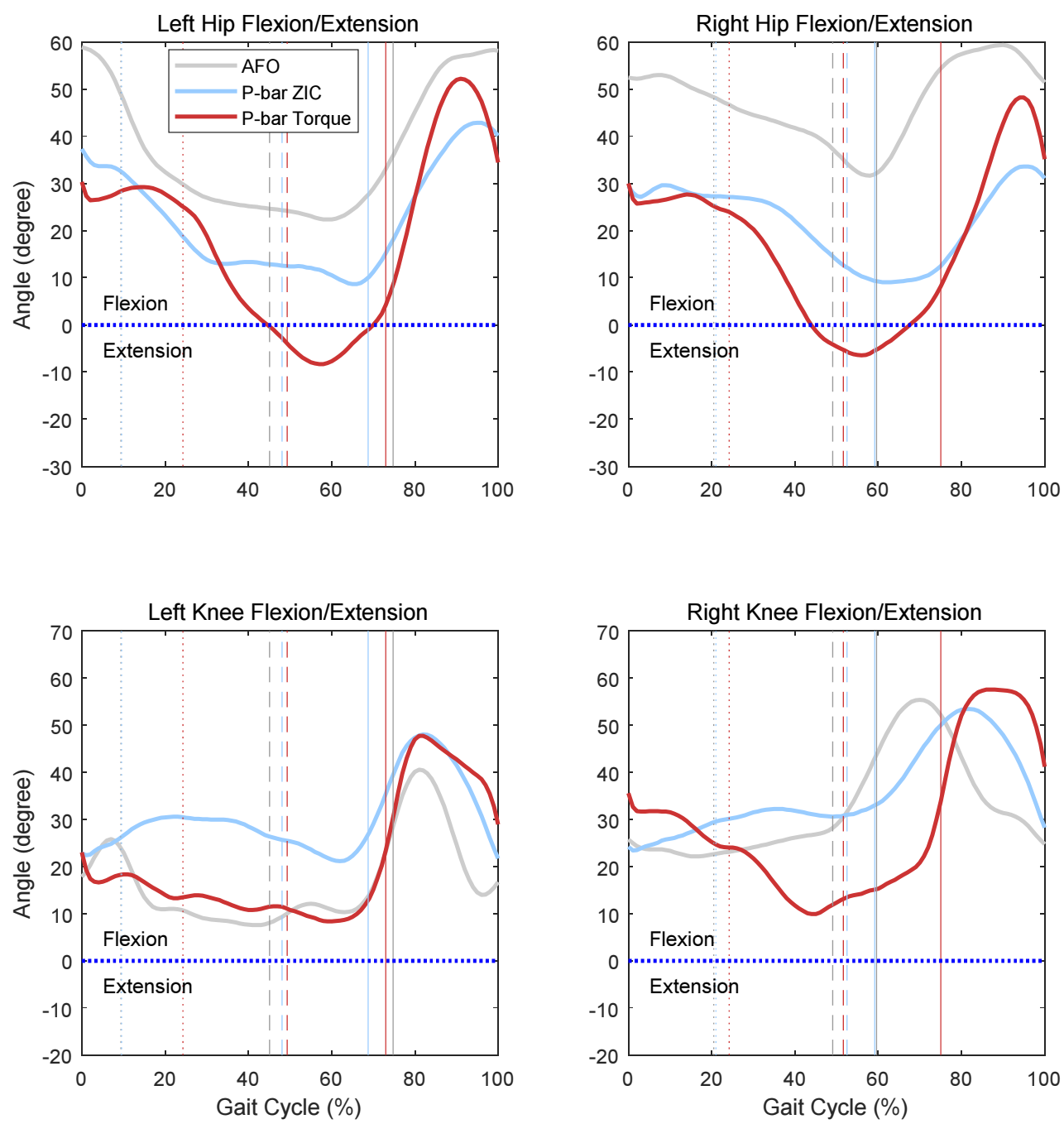

Figure 11. Kinematic result of a patient with spina bifida with Angel Legs and using parallel bars. The solid gray line represents data without Angel Legs, the light blue line represents data with Angel Legs without assistive control, and the red line represents data with assistive control. Vertical lines represents the gait phases. Dotted vertical lines represent the single limb support phase (opposite foot off), dashed vertical lines represent the second double limb support phase (opposite foot contact), and solid vertical lines represent initial swing. 


\subsection{Assistance with a Wheeled Walker}

After three weeks of training, the patient started ground walking with the help of a wheeled walker wearing Angel Legs. The intention detection algorithm was applied, and on each trial, she walked over a track of $20 \mathrm{~m}$ in length (Figure 12).

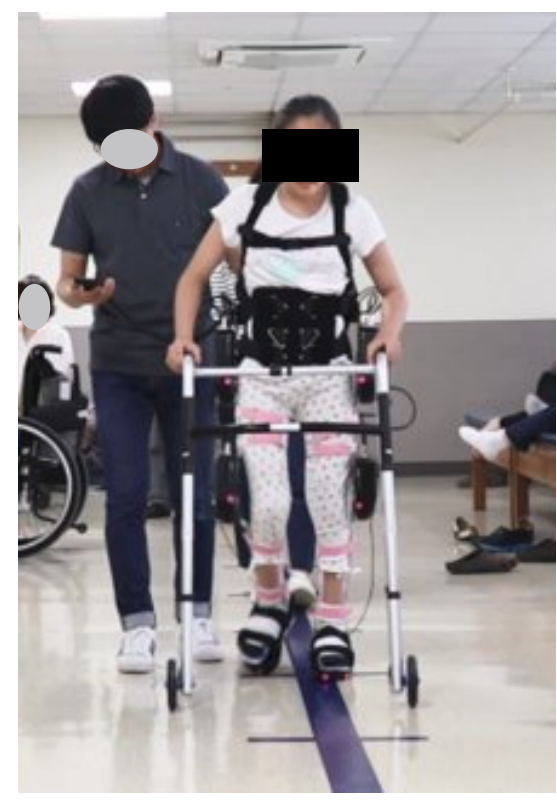

Figure 12. Gait training of a patient with spina bifida with Angel Legs and using a wheeled walker.

Figure 13 and Table 2 show the kinematic results of the hip and knee joints of left and right legs and the gait parameters of a walking trial. The solid lines represent the mean value of the total gait. The gait symmetry index in the stance phase improved from $22.48 \%$ without robot assistance to $4.08 \%$ with torque assistance.

To establish the effectiveness of the torque assistance of the proposed robot, we analyzed the following two cases. The first case was walking with robot assistance using a walker, and the second case was a walking without robot assistance using a walker. The 6-min walking test measured energy consumption by measuring walking distance, oxygen consumption, and heart rate.

According to the results of the 6-min walking test, the walking distances were $62.92 \mathrm{~m}$ and $61.84 \mathrm{~m}$ without and with torque assistance of the proposed robot, respectively. In these two conditions, the average heart rates were very similar: 123.7 and 123.8, respectively. Table 3 shows the results of the oxygen cost analysis: $1.35 \mathrm{~mL} / \mathrm{kg} / \mathrm{m}$ in the no robot support condition and $1.76 \mathrm{~mL} / \mathrm{kg} / \mathrm{m}$ in the robot support condition.

Table 2. Gait parameters of walker gait wearing a torque-controlled robot.

\begin{tabular}{|c|c|c|}
\hline & Left & Right \\
\hline Gait period (s) & 4.11 & 4.08 \\
\hline Stance period (s) & 3.56 & 3.43 \\
\hline Stance period $(\% \mathrm{GC})$ & 86.39 & 86.63 \\
\hline Opposite foot contact ( $\% \mathrm{GC}$ ) & 44.40 & 56.03 \\
\hline Opposite foot off (\%GC) & 27.95 & 42.33 \\
\hline Range of hip motion (degree) & $58.44(-19.62 \quad 38.82)$ & $49.21(-16.47 \quad 32.73)$ \\
\hline Range of knee motion (degree) & $46.91(2.06 \quad 48.97)$ & $57.53\left(\begin{array}{ll}1.89 & 59.42\end{array}\right)$ \\
\hline
\end{tabular}



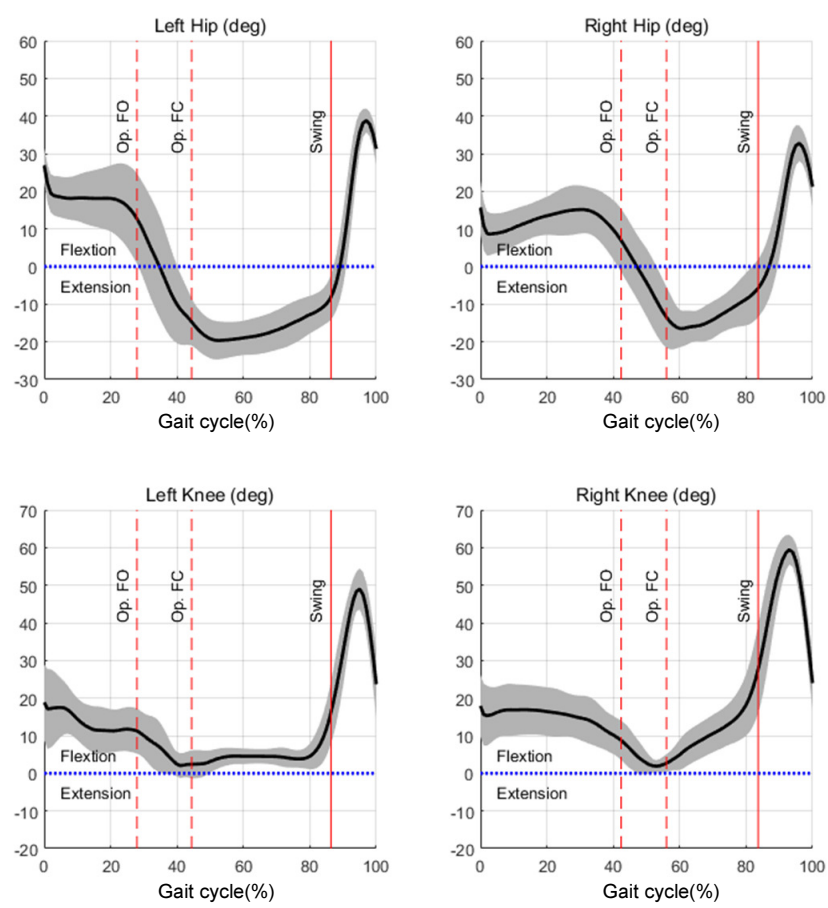

Figure 13. Kinematic result of a walking trial wearing a torque-controlled robotic device and using a wheeled walker. The first vertical dashed lines represent the single limb support phase (opposite foot off), the second vertical dashed lines represent the second double limb support phase (opposite foot contact), and the solid vertical lines represent initial swing.

Table 3. Oxygen costs of the 6-min metabolic test of walking without and with Angel Legs using a wheeled walker.

\begin{tabular}{ccc}
\hline & Without Assistance & Robot Assistance \\
\hline Oxygen cost $(\mathrm{mL} / \mathrm{kg} / \mathrm{m})$ & 1.35 & 1.76 \\
\hline
\end{tabular}

\subsection{Assistance with Forearm Crutches}

In this experiment, bilateral forearm crutches that allowed the patient to use their left and right arms more freely than a walker were used. As shown in Figure 14, the patient wore the proposed robot and used bilateral forearm crutches. Using bilateral crutches, she could stand straighter than using a walker. The bilateral crutches facilitated balance movement between the left and right sides. For five weeks, one hour of the robot walking training using bilateral forearm crutches was conducted every week. After five weeks of training, two 6-min walking tests of walking with robot assistance and without robot assistance were conducted.

Figure 15 shows the results of the five-week gait training. The kinematic results were derived from the mean of $20 \mathrm{~m}$ path walking for each training. The lightest blue line indicates the first weeks' data, and the darkest blue line indicates the fifth weeks' data. The vertical line represents the stance phase of the gait cycle.

As shown in Figure 16a, the stance phase period decreased as the training was repeated weekly, and this may be seen as an increase in walking speed. Figure 16a provides a visual representation of the stance phase period changes and the opposite foot-off period changes. As a result of repeated training, the single limb standing time increased (see Figure 16c).

Table 4 shows the results of the kinematic gait analysis of the motion capture system of robot walking using bilateral crutches. No significant differences between the left and right values of all the gait parameters were observed. The gait symmetry index in stance phase improved from $13.53 \%$ without robot assistance to $-1.10 \%$ with torque assistance, which was similar to the index of the walking of healthy people. Figure 17 shows the results of walking without robot assistance and walking with robot assistance using bilateral crutches. 
In the case of the walking with a torque-controlled robot, the hip joint was fully extended and the range of motion of the knee joint increased.
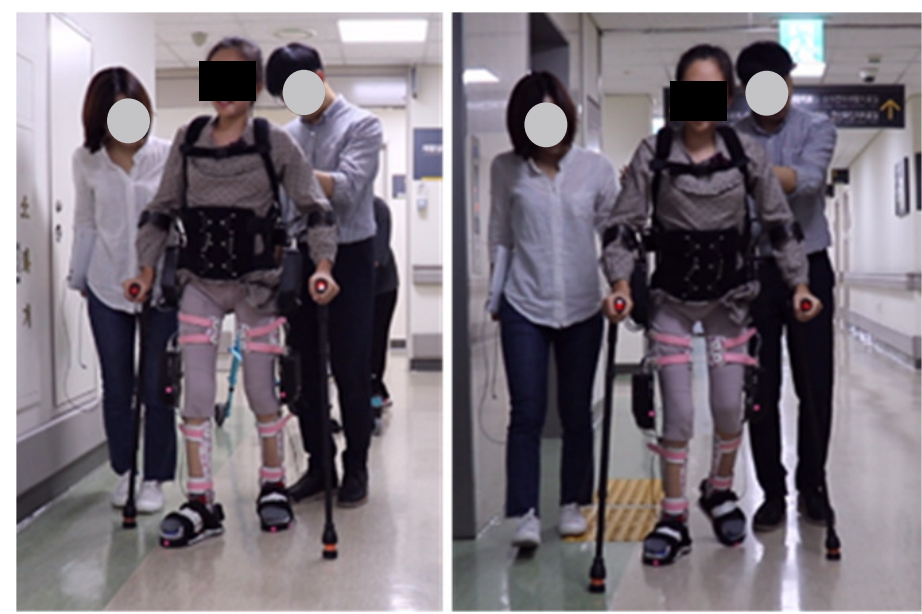

Figure 14. Walking trial of a patient with spina bifida wearing Angel Legs and using bilateral forearm crutches.
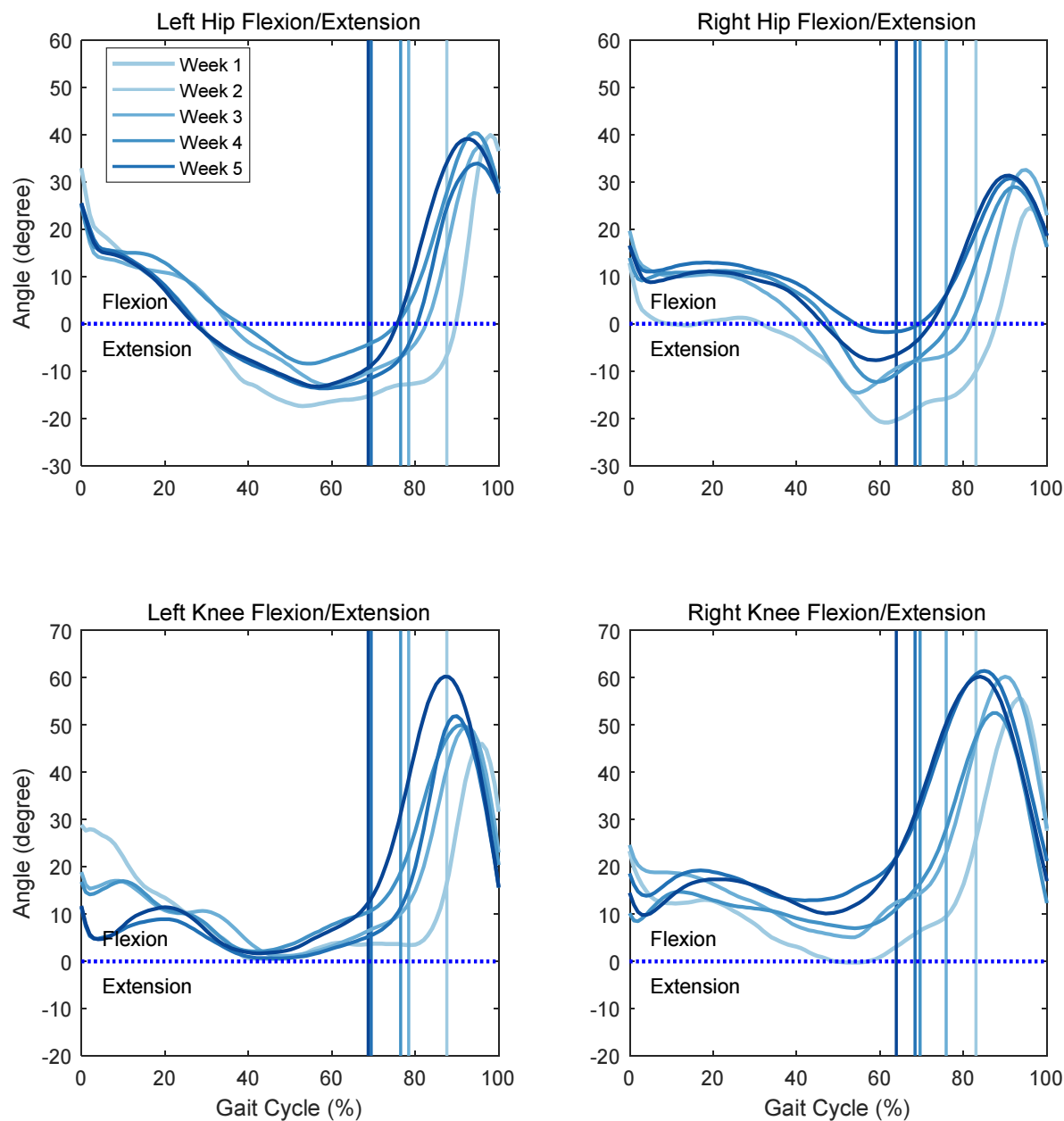

Figure 15. Kinematic results of five-week walking training with Angel Legs and using bilateral crutches. The bright lines represent the early stages of training, and the dark lines represent the later stages of the training. Vertical lines represent the start of swing phase. 


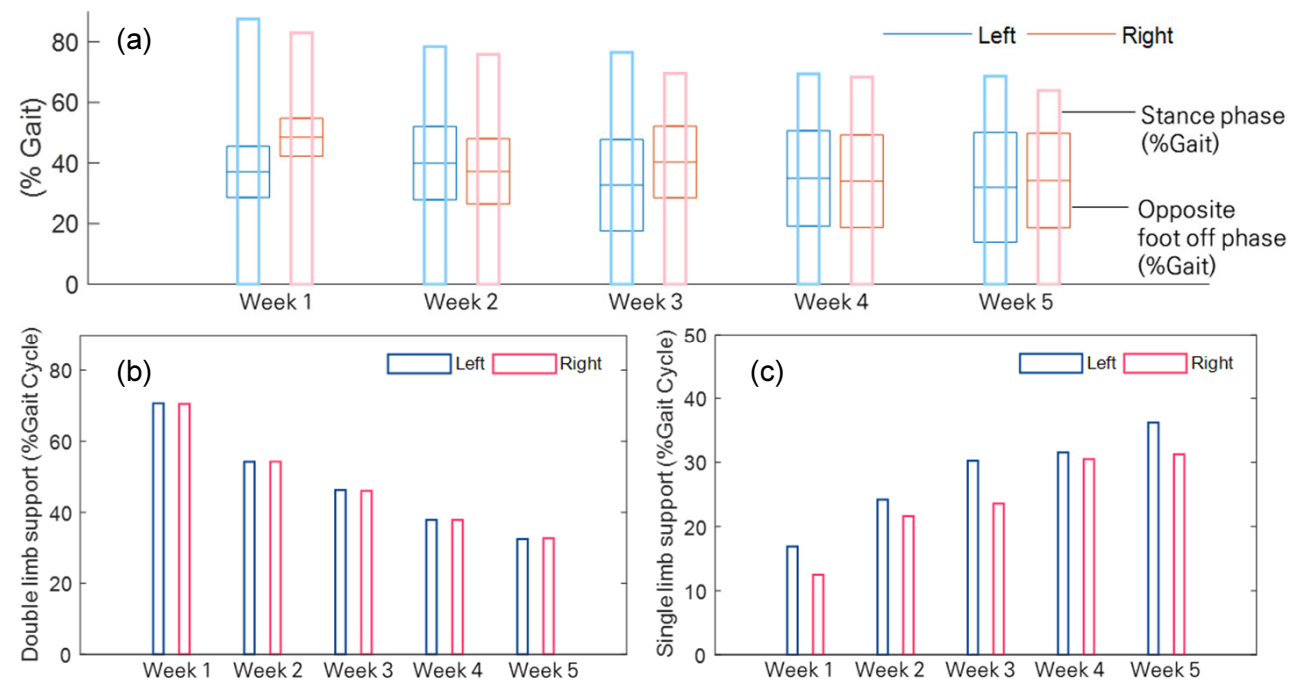

Figure 16. Results of five-week walking training with Angel Legs and using bilateral crutches. (a) Percentage of stance phase and single limb support (opposite foot-off) time of left and right legs. (b) Percentage of double limb support time during a gait. (c) Percentage of single limb support time during a gait.
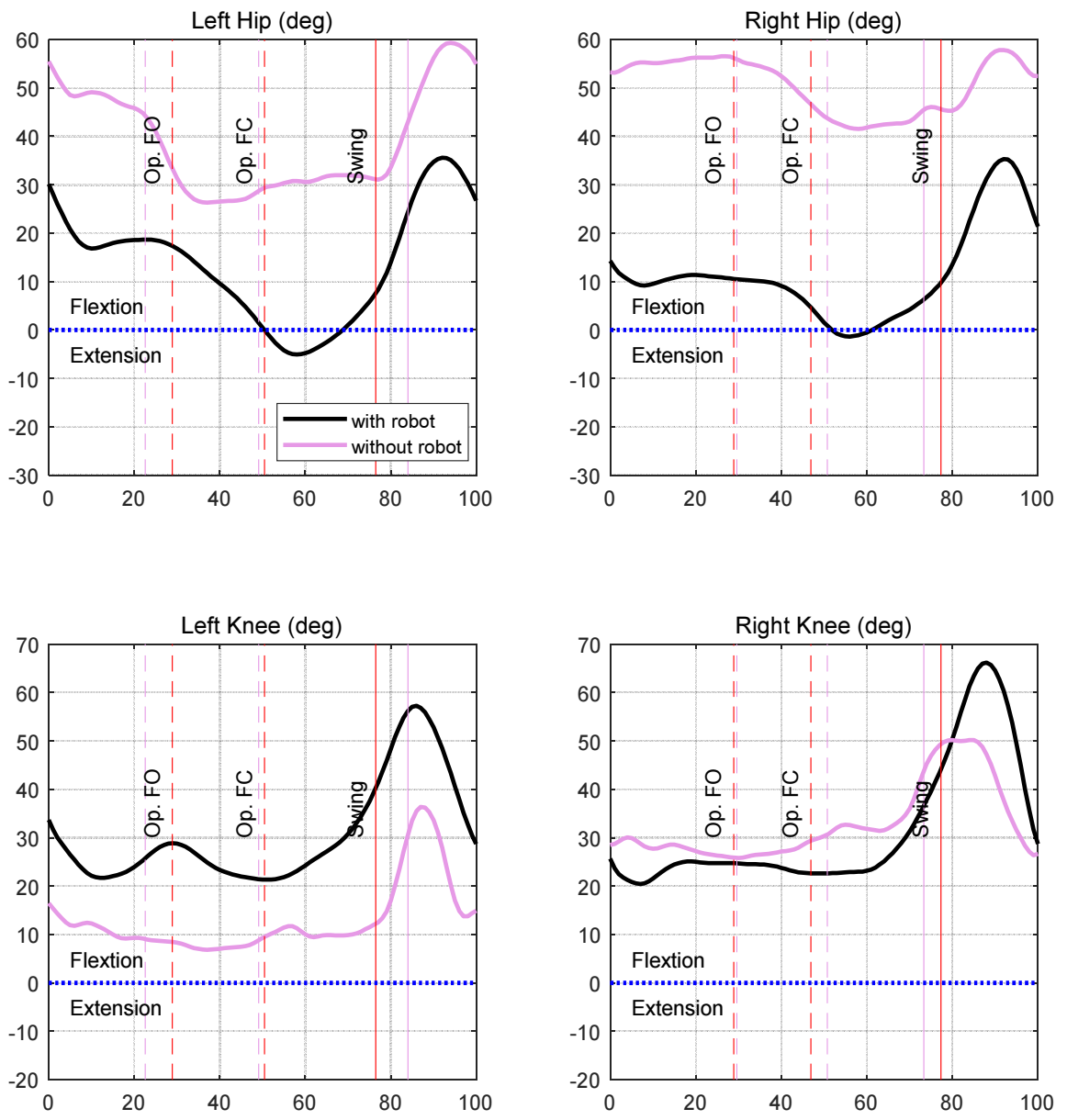

Figure 17. Kinematic results of gait analysis of walking using bilateral crutches. The solid pink line represents data without robotic assistance, and the solid black line represents data with assistance. The first vertical dashed lines represent the single limb support phase (opposite foot-off), second vertical dashed lines represent the second double limb support phase (opposite foot contact), and the solid vertical lines represent the swing phase. 
Table 4. Gait parameters of bilateral crutches gait with Angel Legs.

\begin{tabular}{|c|c|c|}
\hline & Left & Right \\
\hline Gait period (s) & 2.04 & 1.94 \\
\hline Stance period (s) & 1.56 & 1.50 \\
\hline Stance period (\%GC) & 76.47 & 77.32 \\
\hline Opposite foot contact (\%GC) & 50.49 & 46.91 \\
\hline Opposite foot off (\%GC) & 28.92 & 28.87 \\
\hline Range of hip motion (degree) & $40.68(-5.08 \quad 35.59)$ & $36.68(-1.36 \quad 35.32)$ \\
\hline Range of knee motion (degree) & $35.91(21.34 \quad 57.24)$ & $45.77(20.42 \quad 66.19)$ \\
\hline
\end{tabular}

Throughout the 10-week robot walking training, the patient became accustomed to the robot walking and found an optimal walking pattern using the assistive force of the robot. As in Figure 16, the duration of the stance phase decreased with time, similarly to that of the normal gait, which was also associated with an increase in single support time. In addition, the patient's being familiar with walking methods using bilateral crutches and learning the patterns of torque aids reduced her fear of malfunction.

Table 5 shows the oxygen cost results of the 6-min walking test using bilateral crutches. According to the experimental results, after five weeks of training, the energy consumption in the walking with robot assistance was 30\% less than in the walking without robot assistance.

Table 5. Oxygen costs of the 6-min metabolic test of walking without assistance and walking with Angel Legs using bilateral crutches.

\begin{tabular}{ccc}
\hline & Without Assistance & Robot Assistance \\
\hline Oxygen $\operatorname{cost}(\mathrm{mL} / \mathrm{kg} / \mathrm{m})$ & 1.93 & 1.37 \\
\hline
\end{tabular}

\subsection{Assistance with Increasing Knee Extension Assistance}

During the case study, the patient experienced difficulty in weight bearing due to muscle weakness. The extension torque assist of the stance phase and the flexion torque assist of the swing phase were effective in the short- and long-term gait trainings. In order to observe the change in gait patterns according to the magnitude of the extension torque of one side (the weaker side), in particular, the $20 \mathrm{~m}$ path walking tests were performed using different torque profiles on the same training day.

The walking data for three different conditions were collected. As shown in Figure 18, the extension torque of the stance phase of the right knee was different in each condition. In each case, the extension torque level of the right knee was set to $16.5,18.5$, and $20.4 \%$ of normal walking.

Figure 19 shows the kinematic results of the three trials shown in Figure 18. No significant difference in the left and right stance phase periods was observed; however, with an increase of the right knee extension assistive torque, the hip extension range increased.

The results of the analysis of gait parameters are shown in Table 6. In trial 3 and trial 1, the maximum hip extension angles of the left and right were 11.8 and 15.83 degrees greater, respectively. The first double support phase on the right can be considered to indicate the weight support preparation for the left swing. With an increase of the extension torque, the difference between the right and left of the double support phase reduced. 

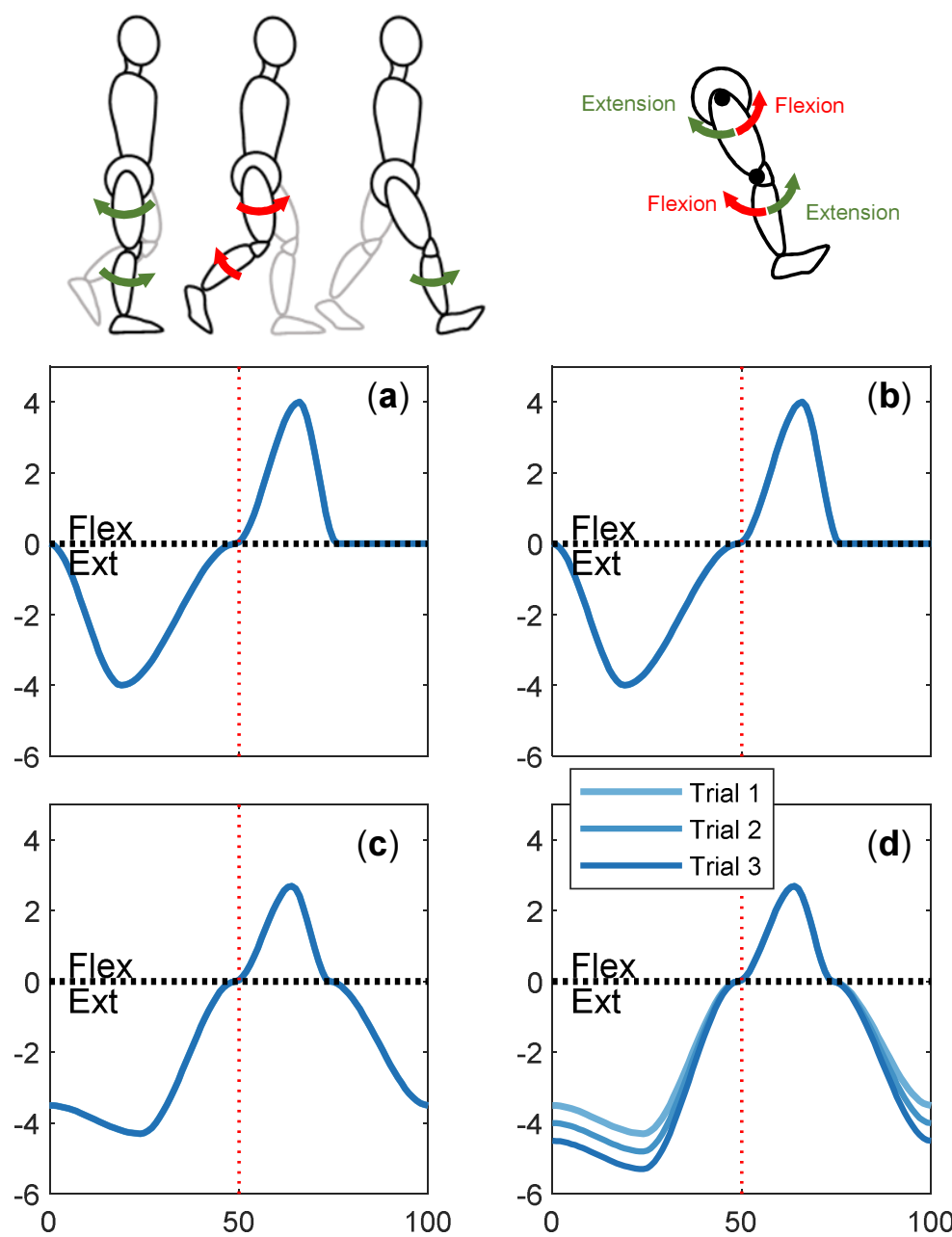

Figure 18. Applied torque profiles of three trials for (a) left hip joint, (b) right hip joint, (c) left knee joint, and (d) right knee joint. Only the extension torques of the right knee joint were different in each case.

Table 6. Gait analysis results of trials.

\begin{tabular}{cccccc}
\hline & & $\begin{array}{c}\text { Without } \\
\text { Robot } \\
\text { Assistance }\end{array}$ & $\begin{array}{c}\text { Robot } \\
\text { Assistance } \\
\text { Trial 1 }\end{array}$ & $\begin{array}{c}\text { Robot } \\
\text { Assistance } \\
\text { Trial 2 }\end{array}$ & $\begin{array}{c}\text { Robot } \\
\text { Assistance } \\
\text { Trial 3 }\end{array}$ \\
\hline Gait cycle (s) & L & 2.75 & 2.28 & 2.10 & 1.93 \\
& R & 2.74 & 2.27 & 2.11 & 1.93 \\
\hline First double support & L & 22.55 & 12.49 & 12.64 & 16.88 \\
(\%Gait) & R & 29.56 & 24.13 & 23.46 & 18.58 \\
\hline Single support & L & 26.55 & 34.18 & 34.45 & 34.30 \\
(\%Gait) & $\mathrm{R}$ & 21.17 & 29.31 & 29.24 & 30.16 \\
\hline Second double support & $\mathrm{L}$ & 34.91 & 24.13 & 23.49 & 18.96 \\
(\%Gait) & $\mathrm{R}$ & 22.63 & 12.41 & 12.91 & 16.93 \\
\hline Stance phase & $\mathrm{L}$ & 84.00 & 70.80 & 70.58 & 70.15 \\
(\%Gait) & $\mathrm{R}$ & 73.36 & 65.87 & 65.61 & 65.68 \\
\hline Maximum hip extension & $\mathrm{L}$ & 26.32 & -5.32 & -14.32 & -17.12 \\
(deg) & $\mathrm{R}$ & 41.58 & -2.08 & -12.06 & -17.91 \\
\hline
\end{tabular}



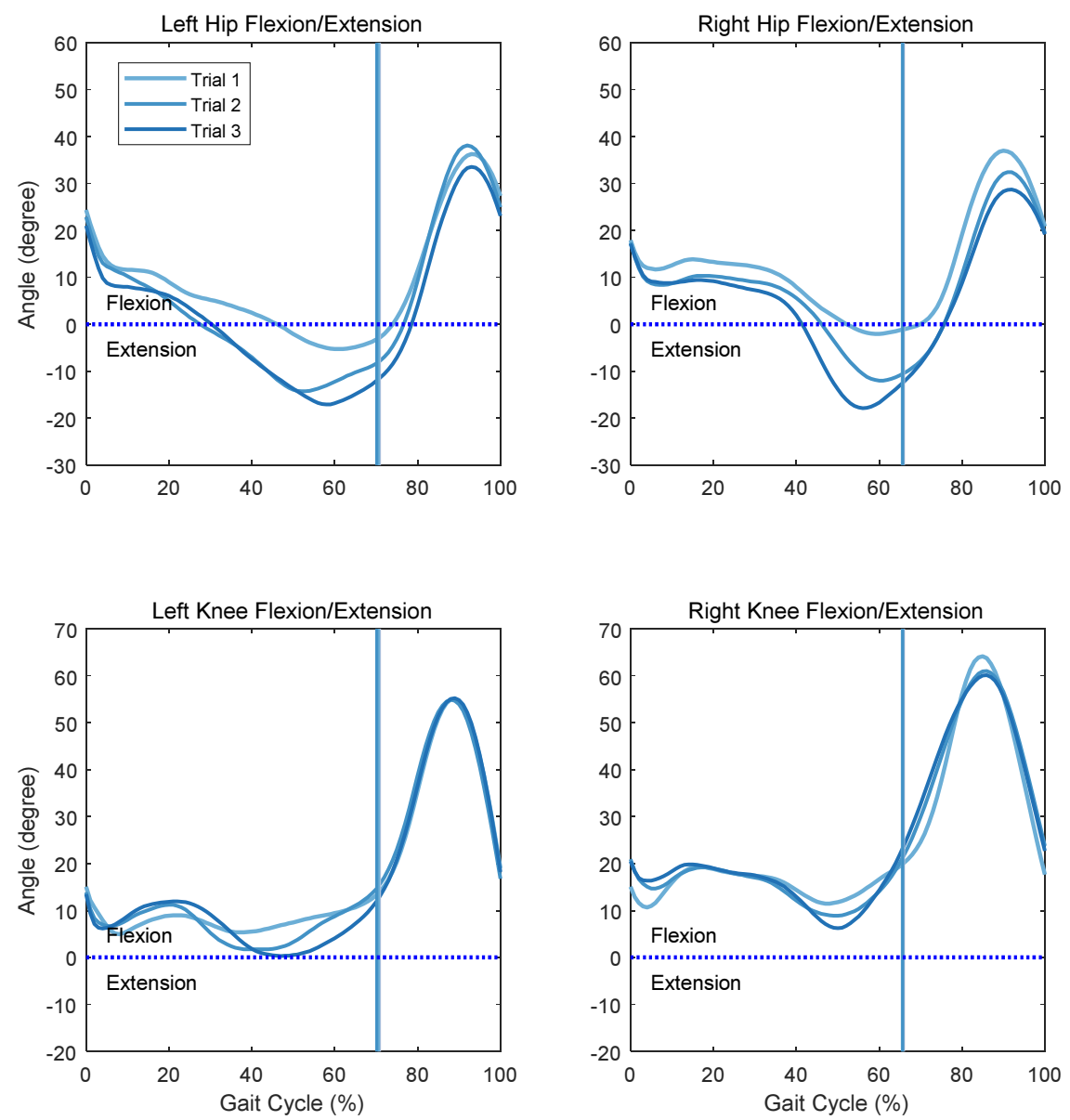

Figure 19. Kinematic results of walking trials with the three different right knee assistive levels.

\section{Conclusions}

In this paper, a method to assist the walking of people with paretic gait using a powered exoskeleton, called Angel Legs, was proposed. First, an algorithm that uses a sensor system to determine the user's real-time walking pattern and intention was proposed. The gait parameters were derived from continuous walking data, and the abnormality of walking was confirmed. Based on an understanding of the gait pattern, control algorithms that provide adequate assistance were also discussed. General equations for assistive torque generation were introduced. These equations can be adjusted for each individual based on personal musculoskeletal characteristics.

To verify the proposed system, a case study with an 11-year-old female child with a discrepancy in leg length and abnormal deformities due to congenital spina bifida was conducted. At the early stage of the training, by using the proposed torque assist robot, the walking symmetry index improved from $22.48 \%$ to $4.08 \%$. At the end stage of the weekly repetitive gait training, the oxygen cost in the 6-min walking test decreased by $29 \%$, from $1.93 \mathrm{~mL} / \mathrm{kg} / \mathrm{m}$ to $1.37 \mathrm{~mL} / \mathrm{kg} / \mathrm{m}$.

Considering that oxygen cost is directly related to gait disability [28], this result demonstrates that gait disability in robot walking improved through training. Through the 10-week robot walking training, the patient grew accustomed to the robot walking and found an optimal walking pattern using the assisting torque of the robot. As shown in Figure 16, the ratio of the stance phase in gait cycle was about $60 \%$, which is similar to that of normal gait. In particular, Figure 15 shows knee flexion in the first double support period of the right leg, i.e., in the loading response section. This characteristic did not appear at the early stage of the training, which shows that the integrated system of the patient and the robot started to use the assist force of the robot in an energy-efficient way. In addition to kinematic and metabolic improvements, the patient's feedback was also 
positive. Before gait training, she did not sense filling of ber bladder; after the training, her urinary function and quality of life improved.

In the near future, robotic gait training proposed in this paper for patients with cerebral palsy who have spastic paralysis will be applied. By increasing the number of cases and patients, the effectiveness of long-term training with regard to various indicators will be examined. Assistive methods for various tasks, such as stair climbing, sitting, and standing, will also be studied.

Funding: There is no funding agency to report.

Institutional Review Board Statement: The study was conducted according to the guidelines of the Declaration of Helsinki and was approved by the Institutional Review Board of Severance hospital, Yonsei university college of medicine (4-2016-0167, approved on 20 April 2018).

Informed Consent Statement: Informed consent for participation was obtained from the parents of the child in this paper according to the rules of the IRB of Severance hospital.

Acknowledgments: The authors would like to thank C. Park for participation in the case study and B. Na for the design and development of the robot hardware.

Conflicts of Interest: The funders had no role in the design of the study; in the collection, analyses, or interpretation of data; in the writing of the manuscript; or in the decision to publish the results. There are no conflicts of interest from the authors.

\section{References}

1. Zorowitz, R.D.; Gillard, P.J.; Brainin, M. Poststroke spasticity Sequelae and burden on stroke survivors and caregivers. Neurology 2013, 80, S45-S52. [CrossRef] [PubMed]

2. Frank, J.S.; Patla, A.E. Balance and mobility challenges in older adults: Implications for preserving community mobility. Am. J. Prev. Med. 2003, 25, 157-163. [CrossRef]

3. Hirvensalo, M.; Rantanen, T.; Heikkinen, E. Mobility difficulties and physical activity as predictors of mortality and loss of independence in the community-living older population. J. Am. Geriatr. Soc. 2000, 48, 493-498. [CrossRef]

4. Bateni, H.; Maki, B.E. Assistive devices for balance and mobility: Benefits, demands, and adverse consequences. Arch. Phys. Med. Rehabil. 2005, 86, 134-145. [CrossRef] [PubMed]

5. Chin, R.; Hsiao-Wecksler, E.T.; Loth, E.; Kogler, G.; Manwaring, S.D.; Tyson, S.N.; Shorter, K.A.; Gilmer, J.N. A pneumatic power harvesting ankle-foot orthosis to prevent foot-drop. J. Neuroeng. Rehabil. 2009, 6, 19. [CrossRef] [PubMed]

6. Onose, G.; Cârdei, V.; Crăciunoiu, Ş.T.; Avramescu, V.; Opriş, I.; Lebedev, M.A.; Constantinescu, M.V. Mechatronic wearable exoskeletons for bionic bipedal standing and walking: A new synthetic approach. Front. Neurosci. 2016, 10, 343. [CrossRef]

7. Lee, H.; Kim, W.; Han, J.; Han, C. The technical trend of the exoskeleton robot system for human power assistance. Int. J. Precis. Eng. Manuf. 2012, 13, 1491-1497. [CrossRef]

8. Contreras-Vidal, J.L.; Bhagat, N.A.; Brantley, J.; Cruz-Garza, J.G.; He, Y.; Manley, Q.; Nakagome, S.; Nathan, K.; Tan, S.H.; Zhu, F.; et al. Powered exoskeletons for bipedal locomotion after spinal cord injury. J. Neural Eng. 2016, 13, 031001. [CrossRef]

9. Holanda, L.J.; Silva, P.M.; Amorim, T.C.; Lacerda, M.O.; Simão, C.R.; Morya, E. Robotic assisted gait as a tool for rehabilitation of individuals with spinal cord injury: A systematic review. J. Neuroeng. Rehabil. 2017, 14, 126. [CrossRef]

10. Louie, D.R.; Eng, J.J.; Lam, T. Gait speed using powered robotic exoskeletons after spinal cord injury: A systematic review and correlational study. J. Neuroeng. Rehabil. 2015, 12, 1-10. [CrossRef]

11. Esquenazi, A.; Talaty, M.; Packel, A.; Saulino, M. The ReWalk powered exoskeleton to restore ambulatory function to individuals with thoracic-level motor-complete spinal cord injury. Am. J. Phys. Med. Rehabil. 2012, 91, 911-921. [CrossRef]

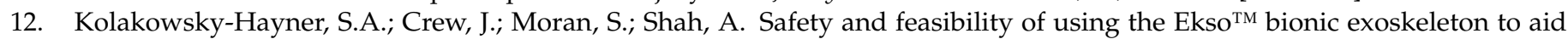
ambulation after spinal cord injury. J. Spine 2013, 4, 003.

13. Quintero, H.; Farris, R.; Hartigan, C.; Clesson, I.; Goldfarb, M. A powered lower limb orthosis for providing legged mobility in paraplegic individuals. Top. Spinal Cord Inj. Rehabil. 2011, 17, 25-33. [CrossRef]

14. Kilicarslan, A.; Prasad, S.; Grossman, R.G.; Contreras-Vidal, J.L. High accuracy decoding of user intentions using EEG to control a lower-body exoskeleton. In Proceedings of the IEEE 2013 35th Annual International Conference of the Engineering in Medicine and Biology Society (EMBC), Osaka, Japan, 3-7 July 2013; pp. 5606-5609.

15. He, Y.; Eguren, D.; Azorín, J.M.; Grossman, R.G.; Luu, T.P.; Contreras-Vidal, J.L. Brain-Machine interfaces for controlling lower-limb powered robotic systems. J. Neural Eng. 2018, 15, 021004. [CrossRef]

16. Simic, M.; Tariq, M.; Trivailo, P. EEG-Based BCI Control Schemes for Lower-Limb Assistive-Robots. Front. Hum. Neurosci. 2018, $12,312$.

17. Suzuki, K.; Mito, G.; Kawamoto, H.; Hasegawa, Y.; Sankai, Y. Intention-based walking support for paraplegia patients with Robot Suit HAL. Adv. Robot. 2007, 21, 1441-1469. [CrossRef] 
18. Veneman, J.F. Exoskeletons supporting postural balance-The BALANCE project. In Replace, Repair, Restore, Relieve-Bridging Clinical and Engineering Solutions in Neurorehabilitation; Springer: Cham, Switzerland, 2014; pp. 203-208.

19. Choi, H.; Na, B.; Kim, S.; Lee, J.; Kim, H.; Kim, D.; Cho, D.; Kim, J.; Shin, S.; Rha, D.W.; et al. Angel-suit: A modularized lower-limb wearable robot for assistance of people with partially impaired walking ability. In Proceedings of the IEEE 2019 Wearable Robotics Association Conference (WearRAcon), Scottsdale, AZ, USA, 26-28 March 2019; pp. 51-56.

20. ReWalk ReStore. Available online: https://rewalk.com/restore/ (accessed on 27 March 2021).

21. Oh, S.; Kong, K. High-precision robust force control of a series elastic actuator. IEEE/ASME Trans. Mechatron. 2017, 22, 71-80. [CrossRef]

22. Lee, C.; Kwak, S.; Kwak, J.; Oh, S. Generalization of Series Elastic Actuator configurations and dynamic behavior comparison. Actuators 2017, 6, 26. [CrossRef]

23. Dos Santos, W.M.; Caurin, G.A.; Siqueira, A.A. Design and control of an active knee orthosis driven by a rotary series elastic actuator. Control Eng. Pract. 2017, 58, 307-318. [CrossRef]

24. Srivastava, S.; Kao, P.C.; Reisman, D.S.; Scholz, J.P.; Agrawal, S.K.; Higginson, J.S. Robotic assist-as-needed as an alternative to therapist-assisted gait rehabilitation. Int. J. Phys. Med. Rehabil. 2016, 4, 370. [CrossRef]

25. Simonsen, E.B.; Cappelen, K.L.; Skorini, R.I.; Larsen, P.K.; Alkjær, T.; Dyhre-Poulsen, P. Explanations pertaining to the hip joint flexor moment during the stance phase of human walking. J. Appl. Biomech. 2012, 28, 542-550. [CrossRef] [PubMed]

26. Alkjær, T.; Larsen, P.K.; Pedersen, G.; Nielsen, L.H.; Simonsen, E.B. Biomechanical analysis of rollator walking. Biomed. Eng. Online 2006, 5, 2. [CrossRef] [PubMed]

27. Robinson, R.; Herzog, W.; Nigg, B. Use of force platform variables to quantify the effects of chiropractic manipulation on gait symmetry. J. Manip. Physiol. Ther. 1987, 10, 172-176.

28. Waters, R.L.; Mulroy, S. The energy expenditure of normal and pathologic gait. Gait Posture 1999, 9, 207-231. [CrossRef] 